\title{
Psychological Intervention for Disorders Stemming from Mass Violence and Genocide: A Case of Kurdish Survivors
}

\author{
Pegah Ali-Mardan Seidi \\ Department of Psychology, College of Education, University of Garmian \\ Email: Pegah.am.seidi@garmian.edu.krd
}

\begin{abstract}
Mass violence and genocide, as human calamities, have always been under scrutiny of scientist and researchers in various fields. Yet, due to the vast irreparable nature of these tragedies, they are still under continues investigation. They are different branches of psychology dealing with these behaviors from different perspective. To this end, the mental disorders of violence Survivors, as well as roots, causes, and conductive factors of mass violence, have been thoroughly studied. Of course, treating and assisting survivors suffered from such kind of violence have been neglected so far. This is more evident in the Middle East and especially Kurdistan, despite various occasions of genocide and mass violence in the region.

Consequently, the present study utilizes a descriptive-analytical approach to review and analyze the existing scientific documents and to introduce treatments for disorders in survivors of genocide and collective violence. To this end, more than 20 research papers and relevant books have been reviewed and analyzed. Results have showed that different approaches like, PET, EMDR, CBT, and TAT were successfully administered to the patients. Finally, in the conclusion section, some therapeutic approaches in accord with socio-cultural condition in Kurdistan are suggested to treat the genocide and collective violence survivors.
\end{abstract}

Keywords: genocide, mental disorder, treatment, Kurdistan.

\section{Introduction}

Mass violence and genocide are destructive realities in history (Staub, 2000) Researchers and scientists, from various tenets, continually investigate roots, dimensions, and consequences of such kind of cruel acts from political, social, historical, and psychological perspectives. Psychology, in particular, plays a vital role in understanding the causes for genocide and providing preventive measures (Munn, 2006). Due to diverse aspects of genocide and its grim and irreparable consequences, this subject is still under the scrutiny of the researchers. Although the UN convention to prevent genocide and to punish genocide 
criminals is a significant preventive tool, it proves to be insufficient in preventing such kind of crimes. This is another reason why research on this issue is so indispensable.

Genocide, or mass slaughter, is derived from "Genos" and "-cide" which mean "race or generation" and "to kill", respectively. This word was firstly coined by Polish jurist, Raphael Lemkin. Based on his opinion, mass slaughter and genocide do not necessarily mean prompt annihilation of a nation or ethnicity, they are schemes designed to annihilate them through a series of diverse destructive measures which destroy foundations of their lives (Mohammadhossenpur, Abastabar \& Qezelselfi, 2015).

'United Nations defined genocide as a crime with the intention to destroy, in whole or in part, a national, ethnic, racial, or religious group. Since then, social scientists and historians have contributed significantly to our understanding of genocide: by explaining its roots, describing its dynamics, and discussing its consequences' (Vollhardt, 2013.p2).

The negative consequences of genocide are not limited to political and social aspects; they affect the prosperity and psychological health of people. The literature shows that such kind of violent acts can lead to severe mental pressure, and such psychological disorders as acute stress reaction, PTSD, anxiety disorder, depression, and bereavement (Ahmed, 2007; Ehntholt, \& Yule, 2006). The issue becomes more important when we stress that negative consequences of genocide are not limited to survivors only, but they also affect the people who committed or observed such kind of violent acts.

Although different branches of psychology have addressed these issues to explore the roots and formation of violent acts, or to illuminate and describe the behavioral and mental trauma in the survivors, few studies are devoted to treatment and assistance of people suffered from such kind of crimes. Lack of studies is more evident in Middle East and esp. Kurdistan, despite the fact that Middle-Eastern people have experienced numerous instances of mass slaughter and genocide at different stages of their history.

Kurds are among the oldest ethnicities leaving in Iran. As a result of Ottoman-Iran wars, a part of Kurdish land was captured by Ottomans. After decomposition of the Ottoman Empire and the World War I, Kurdish land was divided between four countries of Iran, Iraq, Turkey, and Syria. A few Kurds live in Azerbaijan and Armenia, as well (Saniejlal, Moshir Zadeh, Ramazan Zadeh, 2004).This provides a conductive bed for different types of violence against people living in the region. 
Kurds, as the most populated ethnicity lacking an independent country of their own in the Middle East, have suffered various types of mass violence e.g. chemical bombardments of Helebja and Sardasht, Anfal Operations, Dersim, Ghana, and Mar'ash Slaughters, and genocide of Yazidis. Consequently, we expect a higher prevalence of above disorders in survivors suffered from these violent acts in the region. This is attested by studies of particular disorders in general public and survivors of the above calamities. In a study of university students in Kalar, Iraq (one of cities suffered from Anfal Genocide), it was found that most of the students suffered from social-phobia disorder, and its prevalence was comparatively much higher than other countries (Ahmad, Taher, \& Seidi, 2017). In another study, dealing directly with psychological consequences of genocide in Yazidi captive or refuge women during ISIS invasion, it became evident that they suffered from such disorders as PTSD and anxiety disorders (Abbas, 2017). This is the case, while in fact few studies have addressed the issue from treatment perspective.

Based on the above, the present study aims to examine treatment procedures deemed suitable, based on the cultural characteristics of survivors suffered from mass violence and genocide. To this end, we firstly review the findings of recent studies on effects of genocide and mass violence on survivors. Then, we proceed to common treatment procedures for the relevant disorders. Finally, a suitable treatment protocol, based on socio-cultural characteristics in Kurdistan, will be presented.

\section{Methodology}

Desk-research is used to collect data, and a descriptive-analytical method will be used to analyze the data. Consequently, international reports and relevant texts will be studied. This research method was selected, because of the peculiar nature of the subject.

To some extent, this subject requires a specific methodology, and experimental or survey methods are not feasible. Desk-research makes use of literature, as well as, less popular methodologies employed in present psychological studies. There are numerous treatments for survivors suffered from genocide, provided by different psychological tenets like community, clinical and social sub-disciplines. We will proceed with a review of literature, and elaborate on unfathomed aspects of the problem.

\section{Psychological Consequences of Genocide and Mass Violence on Survivors}

The study of people who survived genocide and mass violence include subgroups like direct survivors, children, grandchildren, and the first generation of victim community. 
Majority of first-hand survivors suffer from complications like post-traumatic stress and revenge disorders (Barel, Van Ijzendoorn, \&et al, 2010), somatic symptoms, and disability (Marshall, Schell , and et al, 2005; Hinton, Chhean and et al, 2006 ), sleeplessness, nightmare, nervousness, fatigue, headache, and unpleasant thoughts (Joffe, Brodaty and et al, 2003).

Studies in Halabja, Kurdistan and its neighboring area Chamchamal, attested the longterm consequences of chemical bombardments. The prevalence of miscarriages is fourteen times higher in Halabja, and colon cancer rate is ten times higher in Chamchamal. Many people who did not show chemical-exposure complications after getting in touch with the chemicals, later developed symptoms which were directly traceable to the chemicals. These chemicals could affect human DNAs, and increase the risk of cancer and genetic diseases in the subsequent generations. There is a progressive risk of going blind for people who were directly in touch with the chemical, as well. Other consequences witnessed in the second generation include cleft palates and harelip disorders. The harm caused by chemical attacks is not limited to physiological problems. There are grim psychological complications which affect survivors(Fatehi, 2013).

Sardash in Iran is another city attacked by chemical weapons. Studies attest high rate of depression and anxiety in the region, as well as numerous physiological problems. As a whole, survivors exhibit lower degrees of the mental health (Fathiashtiani, Tavallaee and et al, 2008).

Abbas (2017) in his study "Some Traumas and PTSD among Yazidi Female Survivors from ISIS Army" finds high percentages of trauma in Yazidi female survivors: $38.4 \%$ escaped from ISIS militants, $80.7 \%$ lost at least one family member, $76.9 \%$ were exposed to physical violence, $61.5 \%$ were exposed to sexual violence, $84.6 \%$ were exposed to violence from more than one person, and 75.0\% had PTSD symptoms. Most of the studies witness widespread physiological and mental complications in genocide survivors, but the most prevalent one is PTSD, based the above studies (Sonis, Gibson, 2009; Barel, Van Ijzendoorn, \&et al, 2010).

For instance, in a study by the Transcultural Psychosocial Organization of Cambodia (TPO, 2004), it is found that a vast majority of Democratic Kampuchea's survivors still suffer from some degrees of mental or emotional problems. $81 \%$ of Cambodians experienced violence, 28.4\% suffer from post-traumatic stress disorder (PDST), 11.5\% from mood disorder, and 40\% from anxiety disorder. The US Department of Health and Human Services also studied the mental health problems in regime's survivors living in the United States. It 
was found that $62 \%$ suffered from PTSD, and 51\% demonstrated major depression disorder (the avrage prevalence rates of PTSD and depression in the US are $3.6 \%$ and $9.5 \%$, respectively). There is no wonder if you are aware that $99 \%$ of these Cambodian refugees nearly starved to death, $96 \%$ endured forced labor, $90 \%$ had a family member or friend murdered, and 54\% were tortured (DC-Cam, 2007).

Despite the fact that Middle East is a region with the greatest number of research projects on disorders after incidents (Nemati, Saeedi, and et al, 2012), required treatment needs closer and further investigation. As a result, our study concentrates on elaboration and presentation of a treatment for psychological disorders in genocide survivors.

\section{PTSD}

PTSD includes the overall reactions and symptoms of people facing pressure beyond their possibilities. These disorders are defined by the $5^{\text {th }}$ diagnostic and statistical manual to psychological disorders as a series of specific symptoms people demonstrate after a traumatic or highly stressful event. These criteria include the following: people embody the trauma in their dreams repeatedly, they are not interested in life and avoid the stimuli which remind them of the trauma (e.g. thoughts, emotions, places, people, etc.), unprecedented anxiety and hyper-arousal disorders (including sleeplessness, over-vigilance, lack of concentration, extreme startling responses, anger, lapse of memory regarding the stressful event, dissociation, and defensive avoidance (American Psychiatric Association, 2013).

The above symptoms will last for over a month and will deteriorate their vocational performance ending to disability, economic complications, numerous physiological and psychological disorders like depression, drug-abuse, and severe harm to social and family relations (Nemati, Saeedi, and et al, 2012). Studies confirm a positive correlation between anger \& revenge desire and PTSD \& its intensity (Sonis, Gibson, 2009). They also indicate that aging and lack of treatment intensify the symptoms in patients. Studies on the effect of long-term trauma on survivors claim that passage of time does not benefit the patients since reduction of social supports and aging intensify symptoms like sensitivity, and nightmare in patients (Abhari-Ahmadi, 2000). This fact emphasizes the need for investigating and choosing proper treatment protocols to deal with the disorders.

\section{Specific Treatments for PTSD and Other Disorders Observed in Genocide Survivors}

In countries engaged in war or genocide- due to weakened government and relevant institutions, poor social infrastructures, and drop in psychological hygiene- there are few 
opportunities for regular treatments like personal consultation, group consultation, supportive groups, etc. Consequently, most of the survivors have to tolerate the traumatic symptoms for a long time (Connolly, Sakai, 2011).

World Health Organization (WHO) predicts that psychological disorders will be the second major cause of death and disability in world's population by 2020. Traumas can intensify this alarming statistics and consume national resources, since rendering services to a large number of suffered people can be really costly.

Based on the literature, the following list includes some of the common treatment methods successfully administered to people exposed to traumatic events: Narrative Exposure Therapy (NET), Cognitive Behavior Therapy (CBT), Thought Field Therapy (TFT), Eye Movement Desensitization and Reprocessing (EMDR), Prolonged Exposure Therapy (PET). They will be briefly introduced as follows.

\section{Thought Field Therapy (TFT)}

Thought Field Therapy is a brief treatment, often used as a self-help treatment, developed by psychologist Roger Callahan (Callahan \& Callahan, 2000). Once a specific problem is identified in a client, a typical TFT session begins with exposure to the problem, i.e. the therapist asks the client to think about the problem. The client is asked to think about the problem and this elicits his/her feelings about the matter. Then the client is required to simultaneously stimulate selected acupoints on the surface of the skin, by tapping their fingers on the points in a sequence that is specific to the identified emotion(s). Each TFT tapping protocol or algorithm designates the specific acupoints to be tapped, as well as the order in which they are to be tapped. These algorithms address a range of emotions such as anxiety, fear, anger, shame, embarrassment, and addictive urges. Elements of PTSD, such as hyperarousal, dissociation, and defensive avoidance, are targeted using a trauma treatment protocol. Although there are more advanced levels of TFT that require more extensive training, only the TFT algorithm described above were taught and applied in this study. The scope of this paper will be limited to the use of TFT at the algorithm level to address symptoms of trauma (Connolly and Sakai, 2011).

Sakai and colleagues (2001) summarized clinical applications of TFT at Kaiser Permanente Hospital, Honolulu, Hawaii, with 714 patients. Their findings included statistically significant within-session reductions in self-reported distress with TFT applications for conditions that included anxiety, depression, anger, bereavement, chronic 
pain, panic disorder, and trauma. Sakai, Connolly, and Oas (2010) used TFT in 2006 with 50 adolescents who were orphaned by the Rwandan genocide and were experiencing PTSD symptoms. After one TFT session, participants and caregivers reported significant decrease in PTSD symptoms, including nightmares, flashbacks, bedwetting, depression, feeling of isolation, jumpiness, and concentration difficulty. A one year follow-up showed that these improvements were sustained.

\section{Eye Movement Desensitization and Reprocessing (EMDR)}

Eye Movement Desensitization and Reprocessing therapy is recognized as one of the most influential recent techniques to reduce PTSD symptoms. It includes implosion therapy, cognitive reconstruction, rapid and coordinated eye movements, and bilateral stimulation. This treatment method, introduced by Francine Shapiro (1987), uses behavioral interventions to help clients suffering from post-trauma stress disorder. EMDR has been successfully applied on children, accident survivors, and people who suffer from anxiety, fear, depression, grief, or different types of phobias (Shapiro, 1994).

This model assumes that traumatic event is left unprocessed, since immediate biological reactions to the mental trauma have left it in a neuro-biological isolation (Spector and Read, 1999).The underlying mechanism for this therapy is to lead the unprocessed information to an acceptable synchronization level. The hypotheses proposed for the underlying mechanisms of this treatment are derived from empirical findings about limbic system, neurobiology, trauma therapy, and sleep during rapid eye-movement stage. Treatment process is relatively fast, unlike traditional psychological treatments, and therapy sessions are not long. Treatment process invites the patient to imagine a similar traumatic situation, hold the image in mind and continuously follow the gentle transversal movements the therapist makes with his/her finger or an object. The process will last for a couple of minutes until the patient reports that the image is less traumatic.

It is believed when patients experience deep relaxation, they are able to cognitively reconstruct the traumatic event and reduce the symptoms. Then, the therapist asks the patient to review and describe all negative thoughts occupying his/her mind. Next, the patient is required to trace the movements therapist makes with a finger or another moving object and think positively. The patient follows the movement as long as s/he is able to retain the positive thoughts in mind. This therapy consists of eight stages, based on a combination of exposure therapy and cognition. The number of sessions required for each stage and number 
of stages in each session, as well as the required time for each session depend on client's condition and the intensity of trauma (American Medical Association, 2009).

Empirical evidence suggests that it is one of the most influential treatments for posttraumatic stress disorders. It is more effective in treatment of traumas than other types of therapies (Rothbaum, Astin, \& Marsteller, 2005; Power, McGoldrick \& et al, 2002). Researchers claim that Eye Movement Desensitization and Processing proves satisfactory in people who suffer from guilt, fear, depression, annoying thoughts, and anxiety commonly caused by traumatic experience (Gorman, Battista, 1998).

\section{Cognitive-Behavior Therapy (CBT)}

Cognitive-Behavior Therapy is a combination of behavioral therapy and cognitive one whether in cognitive therapy or in cognitive psychology frameworks. It is a result of evolution in behavioral treatment. This approach, nowadays, includes relatively different theories and perspectives which only share the importance of cognitive processes as a medium for data processing and personal reactions to stimuli. This approach makes use of terminology and concepts which are meaningful and measurable in a behavioral framework.

Its effectivity in reduction of PTSD symptoms has made it the first choice to treat such kind of patients. Patients susceptible to suicide and psychosis can benefit from this therapy, as well (Bisson, Ehlers, 2007). The patient firstly learns to handle his/her emotions and tensions caused by annoying memories. S/he will learn to relax and soothe the pains. Then, the therapist helps the patient to encounter annoying memories and describe the happening. Repetition reduces the unpleasant feeling and the patient will feel more relaxed. Finally, the therapist trains the patient to alter his/her negative thoughts and organize the life, based on the above (Jennings, 2008).

In literature, a great part is devoted to cognitive-behavioral approaches, as the first choice to treat PTSD disorders in children and young adults (Donnelly, Amaya-Jackson, 2002). The results of several controlled random studies by Foa et al. (2006) show that cognitive-behavioral therapy is an effective treatment for PTSD stress. Another study shows that early treatment improves the effectivity of the above method (Darharaj, Moradi \& et al, 2015).

\section{Prolonged Exposure Therapy (PET)}

Prolonged Exposure Therapy is one of cognitive-behavioral methods to treat PTSD stress disorder. This method aims to encourage people suffering from avoidance encounter 
their fears and anxieties (Tull, Gratz, \& et al 2004). In this method patient describes the details of his/her horrifying experiences under the supervision of a therapist. S/he safely encounters the horrifying stimulus either mentally or in the real environment to reduce his/her anxiety. Exposure will be effective and safe if it is directly guided by the therapist, so that the meaning of events for patients can be altered, and the conditioned responses can be turned off (Foa \& Meadows, 1997).

Turk et al. (2011) reported this treatment as effective, studying patients from Afghanistan and Iraq. Powers et al. (2010), in their meta-analysis of 13 controlled studies with quasi-experimental and control groups using mental and real exposure therapy, attested that it is more effective than other methods in treatment of PTSD patients including soldiers, rape victims and survivors of natural and unnatural accidents.

Based on the literature, medical treatment is not effective in treatment of PTSD disorders. Such kinds of treatments are inefficient in treatment of disorders because of their symptom-oriented property, and long-term consumption of drugs may cause side-effects. These psychological treatments do not cause any side-effects and play a crucial role in curing the disorders. These techniques are problem-oriented and are supported by considerable theoretical and empirical studies to deal PTSD disorder (Abhari-Ahmadi, 2000).

\section{Discussion and Conclusion}

According to the literature, it can be concluded that genocide and mass violence impose grim consequences to the whole society, including psychological ones. These consequences directly and indirectly affect the people who were engaged in violence. Although the impact of incidents varies based on psychological differences in people, there are increased risks of disorders like PTSD and nervous disorders (anxiety and depression) in case of extreme conditions. Sometimes these disorders are continued and chronic. They can even lead to mood disorders. Thus, early treatment is compulsory to prevent subsequent complications.

Health is deemed as one of the basic human rights and some countries are committed to prevent the diseases and to take care of citizens' well-being (World Health Organization, 2008), but there are other countries which are unable to take any effective measures in this regard, due to war or violence. These countries either lack required personnel for psychological treatments or the existing personnel lack the needed training and specialty to render services. 
Currently, there are many centers in Kurdistan which host projects to specifically treat the Halabja chemical attack survivors. An example is the Zakho Emergency Hospital and Trauma Center which was established in 2009 financed by the Economic Support Funds (ESF) of the United States. The trauma center includes surgical wards, MRI, and X-Ray facilities. The Kirkuk center for tortured victims has a specialized survivor project called the Halabja Center for Victims of Chemical Attacks which offers psychological and medical treatments for chemical weapons victims and their families. The KRG is also working with the World Health Organization to set up a psychological services, and a child protection center. Trauma assistance and timely therapy and medical treatment are crucial in the recovery of victims, even 30 years after the incident.

Although fruitful measures are taken to treat physiological and psychological disorders in survivors of chemical bombardments, identification and treatment of psychological disorders is a must even for the second and third generations, due to their longterm consequences. Rendering any type of psychological treatment should be based on sociocultural texture and characteristics. These services should be provided with a reasonable cost.

Based on the above, our proposed treatment for Kurdish genocide survivors is a combination of mentioned treatment programs with other types of programs, e.g. spiritual therapy. Some of the treatments are not in line with cultural features of the people. For example, Yazidi Kurds are very introvert. They are not willing to elaborate on their memories and emotions. Middle-Eastern people and esp. Kurds are very religious, consequently the treatment protocols mentioned earlier may not suffice to treat them. A combination of these approaches with spiritual therapy can simultaneously provide them with benefits from various approaches. Some of the studies show that faith and religious beliefs can considerably alleviate their traumas, and lead them to easier acceptance of stressful events (Mirzamani, 2004).

In a nut shell, it can be stated that therapists nowadays use hybrid measures to treat disorders. This is particularly true for PTSD patients who need more serious treatment and supportive measures (Gorman, Battista, 1998).Using a combination of treatment measures helps to determine the particular treatment helpful for a certain patient. The combined treatment methods at their best, provide a systematic and effective treatment protocol.

Prognosis and psychological treatments are required to prevent transition of disorders to offspring and future generations. Preventive psychological measures to decrease the 
intensity of psychological disorders stemming from violence and traumatic events can be taken on three different levels:

The first level includes identifying and training the people who may encounter traumatic events. The second level includes various psychological treatments which should be rendered immediately after traumatic incidents. The third level deals with PTSD and relevant disorders (Mirzamani, 2004).

In the present study, we faced some limitations and barriers due to the research method, and insufficient studies on Kurdish genocide survivors. Kurdish speaking lands are so vast and located in different countries. Lack of a Kurdish central government, differences in facilities, and cultural or behavioral characteristics make generalization from our findings difficult. Therefore, we recommend future researchers to concentrate on genocide survivors in various Kurdish-speaking regions. Researchers are encouraged to study the prevalence of other types of disorders, and to recommend proper treatments.

\section{References}

Abbas, NQ. (2017). Introducing some trumas and PTDS among Yazidi female survivors from ISIS army. Genocide of Kurdish people from a scientific viewpoint , $4^{\text {th }}$ Scientific Conference in College of Education, University of Garmian.

Abhari-Ahmadi, SA. (2000). Long-term effects of war on psychosocial status of patients with post-traumatic stress

Ahmad, RJ., Taher, BH., Seidi, PA. (2017). Prevalence of Social Anxiety in Students of college of Education - University of Garmian, $4^{\text {th }}$ Global Conference on Psychology, Educational Science, Law and Social Science. Shiraz, Iran.

Ahmed, A.S. (2007). Post-traumatic stress disorder, resil- iency and vulnerability. Advances in Psychiatric Treat- ment, 13, 369-375.

American Psychiatric Association (2013). Diagnostic and statistical manual of mental disorder (5th. Ed) Washington Dc: American Psychiatric Association.

Barel, E., Van IJzendoorn, M. H., Sagi-Schwartz, A., \& Bakermans-Kranenburg, M. J. (2010). Surviving the Holocaust: A meta-analysis of the long-term sequelae of a genocide. Psychological Bulletin, 136, 677-698.

Bisson, JI., Ehlers, A., Matthews, R., Pilling, S., Richards, D., Turner, S. (2007) Psychological treatments for chronic post-traumatic stress disorder. Br J Psychiatry. 190(2):97-104. 
Callahan, R. J., \& Callahan, J. (2000).Stop the nightmares of trauma. Chapel Hill, NC: Professional Press.

Connolly, S., Sakai, C. (2011). Brief Trauma Intervention with Rwandan GenocideSurvivors Using Thought Field Therapy. International Journal of Emergency Mental Health, Vol. 13, No. 3, pp. 161-172.

DC-Cam, (2007). Coping with the Psychological Trauma of the Khmer Rouge. Avallble from:

http://www.genocidewatch.org/images/Cambodia_29_May_07_Coping_with_the_Psych ological_Trauma_of_the_Khmer_Rouge.pdf.

disorder. Tehran; Proceedings of the Third Symposium of Neurological Complications Psychological War.

Donnelly CL, Amaya-Jackson L. (2002). Posttraumatic Stress Disorder in Children and Adolescents: Epidemiology, Diagnosis and Treatment Options. Pediatr Drugs. 4: 159170.

Ehntholt, K.A., \& Yule, W. (2006). Practitioner review: Assessment and treatment of refugee children and adoles- cents who have experienced war-related trauma. Journal of Child Psychology and Psychiatry, 47, 1197-1210.

Emdria.org, Chicago: American Medical Association; c1999-2009 [updated 2009 Apr 19; cited 2008 Nov 6]. Available from: http://www.emdria.org

Fatehi, T. (2013). The health effects of the Halabja genocide, 25 years on. Medya Magezin. Available from: medyamagazine.com/the-health-effects-of-the-halabja-genocide-25years-on/.(June 2017)

Fathiashtiani A., Tavallaee S. A., Azizabadi Farahani M., Moghani lankarani M. (2008). Association of Psychological Symptoms and Self Esteem in Chemical warfare agent Exposed Veterans. Journal of Military Medicine, 9(4): 273-282

Foa, EB., Meadows, EA., (1997). Psychosocial treatments for posttraumatic stress disorder: a critical review. Annu Rev Psychol. 48: 449-80.

Foa. E.B (2006), Psychosocial Therapy for PTSD, Jornal of clinical Psychiatry, Supple 2, 67, Page 40-45

Gorman, JM., Battista, D., Goetz, RR., Dillon, DJ., Liebowitz, MR., Fyer, AJ., et al. (1989). A comparison of sodium bicarbonate and sodium lactate infusion in the induction of panic attacks. Arch Gen Psychiatry 46: 145-50. 
Hinton DE, Chhean D, Pich V, Hofmann SG, Barlow DH. Tinnitus among Cambodian refugees: relationship to PTSD severity. J Trauma Stress. 2006;19 (4):541-546.

Jennings SA. (2008). Randomized controlled trial of cognitivebehavioral treatment for posttraumatic stress disorder in severe mental illness. J Consult Clin Psychol.76:259- 71.

Joffe, C., Brodaty, H., Luscombe, G., \& Ehrlich, F. (2003). The Sydney Holocaust study: Posttraumatic stress disorder and other psychological morbidity in an aged community sample. Journal of Traumatic Stress, 16, $39-47$.

M. Darharaj, A.R. Moradi, J. Hasani, A. Ghaednia Jahromi (2015). Effectiveness of Cognitive Behavioral Therapy on the Reduction of Pathological Symptoms of Posttraumatic Stress Disorder in Adolescents. Alborz University of Medical Science. 4(3): 168-175

Marshall GN, Schell TL, Elliott MN, Berthold SM, Chun CA. Mental health of Cambodian refugees 2 decades after resettlement in the United States. JAMA. 2005;294(5):571-579.

McLeigh, J. D. \& Sianko, N. (2011) What should be done to promote mental health around the world? American Journal of Orthopsychiatry, 18, 83-89.

Mirzamani, SM. (2004). Principles of Psychological Intervention in War Zones, Journal of Military Medicin. 6(3): 217-223.

Mohammadhossenpur, Kh., AbastabarR., Qezelselfi, MT. (2015). Anfal (Kurdish genocide in Iraq) and modernity. Master's thesis in political science, Mazandaran University.

Munn, S. (2006). Dialogue Toward Agenocide: Encountering the Other in the Context of Genocide. Journal of Humanistic Psychology, 46(3), 281-302.

Nemati, Sh., Saeedi, M., Marzieh SM., Ezzadi, H. (2012). The Psychological Consequences of the Iraq War Against Iran. Holy Defense Research Center, 1 (2), 81-95.

Tuerk PW1, Yoder M, Grubaugh A, Myrick H, Hamner M, Acierno R. (2011). Stress Disorder: An Examination of Treatment Effectiveness for Veterans of the Wars in Afghanistan and Iraq. J Anxiety Disord. 25(3): 397-403.

Power, K., McGoldrick, T., Brown, K., Buchanan, R., Sharp, D., Swanson, V., \& Karatzias, A. (2002). A controlled comparison of eye movement desensitization and reprocessing versus exposure plus cognitive restructuring, versus waiting list in the treatment of post traumatic stress disorder. Journal of Clinical Psychology and Psychotherapy, 9(5), 299318.

Powers, MB., Ferenschak, MP., Halpern, JM., Foa, EB.(2010). A meta-analytic review of prolonged exposure for posttraumatic stress disorder. Clin Psychol Rev. 30(6):635-41. 
Rothbaum, B.O., Astin, M.C., \& Marsteller, F. (2005). Prolonged exposure versus eye movement desensitization and reprocessing (EMDR) For PTSD rape victims. Journal of Traumatic Stress, 18, 607-616.

Sakai, C., Connolly, S., Oas, P. (2010). Treatment of PTSD in Rwanda genocide survivors using Thought Field Therapy. International Journal of Emergency Mental Health,12(1), 41-49.

Sakai, C., Paperny, D., Matthews, M., Tanida, G., Boyd, G., Simons, A.,..., \& Nutter, L. (2001).Thought Field Therapy clinical applications: Utilizing an HMO in behavioral medicine and behavior health services. Journal of Clinical Psychology, 57, 1215-1228.

Saniejlal, M., Moshir Zadeh, H., Ramazan Zadeh, A. (2004). Investigating the causes of Kurdish internationalization in the Middle East. Master's thesis in International relations, Tehran University.

Shapiro F. Alternative stimuli in the use of EMD (R). J Behav Ther Exp Psychiatry 1994; 25: 89.

Sonis, J., Gibson, JL., de Jong, JT., Field, NP., Hean, S., Komproe, I. (2009). Probable posttraumatic stress disorder and disability in Cambodia: associations with perceived justice, desire for revenge, and attitudes toward the Khmer Rouge trials. The Journal of the American Medical Association. 5;302(5):527-36.

Spector J, Read J. (1999). The status of Eye Movement Desensitization and Reprocessing (EMDR). Psychotherapy. 6:165-74.

Staub, E. (2006). Reconciliation after Genocide, Mass Killing, or Intractable Conflict: Understanding the Roots of Violence, Psychological Recovery, and Steps toward a General Theory. Political Psychology, 27(6), 867-894.

Tull, M., Gratz, Lk., Salters, MK., Roemer, L. (2004). The Role of Experiential Avoidance in Posttraumatic Stress Symptoms and Symptoms of Depression, Anxiety, and Somatization. Journal of Nervous \& Mental Disease: 192 (11) : 754-761.

Vollhardt, JR., \& Bilewicz, M. (2013). After the Genocide: Psychological Perspectives on Victim, Bystander, and Perpetrator Groups. Journal of Social Issues, 69( 1) 1-15.

World Health Organization.(2008). mental health gap Action program: scaling upcare for mental. neurological and substance use disorder. Geneva, Switzerland. 